\title{
Adding mode choice to multiagent transport simulation
}

\section{Journal Article}

Author(s):

Rieser, Marcel; Grether, Dominik; Nagel, Kai

Publication date:

2009

Permanent link:

https://doi.org/10.3929/ethz-b-000080697

Rights / license:

In Copyright - Non-Commercial Use Permitted

Originally published in:

Transportation Research Record 2132, https://doi.org/10.3141/2132-06 


\title{
Adding Mode Choice to Multiagent Transport Simulation
}

\author{
Marcel Rieser, Dominik Grether, and Kai Nagel
}

It had been shown previously that so-called agent-based traffic microsimulations could be used for dynamic traffic assignment, that is, iterative route adjustment, until either a Nash equilibrium or some steady state distribution between alternatives had been found. It was also shown that the same approach could be extended to (departure) time adjustment; that is, time adjustment and route adjustment could exist in the same iterative approach. In this paper it is shown that the approach can be extended to mode choice by forcing every synthetic traveler to consider every available mode. The implementation is verified with a test case for which an approximate solution can be analytically derived and for which it is shown that simulation and theory are consistent. It is then applied to a large-scale real-world example, the metropolitan Zurich, Switzerland, area, with about 1 million inhabitants. For this example, it is shown that the adaptive scheme, albeit seemingly simple, can outperform a more traditional approach that first computes mode choice on the basis of aggregate data and then runs the assignment for car traffic only. Sensitivity tests show that the model reacts in meaningful ways, in particular concerning the interaction between the time structure of activities and mode choice.

The still increasing volume of traffic asks for different, mature measures. It is generally accepted that just building new roads is not a sustainable way out of this problem, but that the amount of traffic needs to be regulated, or alternative modes of transportation need to be used. This makes traffic forecasts more and more complex, as the proposed measures also gain in complexity. As an example, timeand vehicle-dependent road pricing schemes could be mentioned [e.g., Verhoef (1) and Bonsall et al. (2)].

The traditional four-step process [see, e.g., Ortúzar and Willumsen (3)] has some shortcomings with respect to such questions, because neither time-dependent (such as time-variable toll) nor mode choice problems are adequately addressed. Mode choice is traditionally approached by so-called trip-end (after the trip generation step) or trip-interchange (after the trip distribution step) models. Trip-end models suffer from the obvious shortcoming that the accessibility of the trip destination by mode is completely irrelevant. Trip-interchange models are better, but they neglect the possible correlation between destination and mode choice. In consequence, models of simultaneous mode and destination choice were developed [e.g., Richards and

Transport Systems Planning and Transport Telematics, Berlin Institute of Technology, D-10587 Berlin, Germany. Alternate address for M. Rieser: Institute for Transport Planning and Systems ETH Zurich, CH-8903 Zurich, Switzerland. Corresponding author: M. Rieser, rieser@vsp.tu-berlin.de and rieser@ivt.baug.ethz.ch.

Transportation Research Record: Journal of the Transportation Research Board, No. 2132, Transportation Research Board of the National Academies, Washington,

D.C., 2009, pp. 50-58.

DOI: $10.3141 / 2132-06$
Ben-Akiva (4), Jonnalagadda et al. (5), and Boyce and Bar-Gera (6)], sometimes on the basis of more traditional trip modeling, sometimes in the context of activity-based demand modeling. VISEVA is a software version of a simultaneous destination and mode choice model (7).

However, all these models eventually produce origin-destination (O-D) matrices, which are then fed into the assignment procedure. There is at least one O-D matrix for the car mode, and another matrix for the non-car mode. Often these days, these matrices are timedependent, that is, there are different such matrices for different time slices. These O-D matrices are then assigned to the network, where quite sufficient public transit assignment routines have been developed [e.g., Nielsen and Frederiksen (8) and Nökel (9)].

Unfortunately, however, these assignment models at the downstream end of the procedure seem to be a bit removed from the demand generating behavioral framework farther upstream. For example, it seems that congestion effects need to be manually integrated by taking an impedance matrix from the assignment and using it for the generalized cost functions in the mode choice model. Similarly, small-scale effects such as local accessibility cannot be represented. Any timedependent reaction, such as possible earlier departure in the morning because of a reduced service frequency in the early evening, seems to be difficult to represent.

In this situation, microscopic, behavior-based simulations may be applied to research the outcome of proposed measures [e.g., Zhang and Levinson (10)]. Yet, such models usually are limited to small scenarios for performance reasons. But as the environmental aspects gain higher attention, the demand rises for behavior-based simulations that support large-scale scenarios as well as alternative transportation modes besides private cars. In this paper a description is given of how a large-scale microscopic car-only simulation was extended to also handle non-car modes, such as public transit. Especially, the modechoice reaction of the simulated agents is compared with the mathematically to-be-expected reactions, to verify the correct functioning of the extended simulation. Afterward, the mode choice model is applied to a large-scale application to test its feasibility in a real-world context.

\section{SIMULATION STRUCTURE}

\section{Overview}

Each traveler of the real system is modeled as an individual agent in this simulation. The overall approach consists of three important pieces:

- Each agent independently generates a so-called plan, which encodes the agent's intentions during a certain time period, typically a day. 
- All agents' plans are simultaneously executed in the simulation of the physical system. This is also called the traffic flow simulation or mobility simulation.

- There is a mechanism that allows agents to learn. In this implementation, the system iterates between plans generation and traffic flow simulation. The system remembers several plans per agent and scores the performance of each plan. Agents normally choose the plan with the highest score, sometimes reevaluate plans with bad scores, and sometimes obtain new plans by modifying copies of existing plans.

The simulation approach is the same as in many of the authors' previous papers [e.g., Raney and $\operatorname{Nagel~(11,~p.~42)]:~}$

A plan contains the itinerary of activities that the agent wants to perform during the day, plus the intervening trip legs the agent must take to travel between activities. An agent's plan details the order, type, location, duration, and other time constraints of each activity and the mode, route, and expected departure and travel times of each leg. A plan can be modified by various modules. In the test scenario, the time adaptation module is used; the large-scale application additionally uses a router module. The time adaptation module changes the timing of an agent's plan. A very simple approach is used that just applies a random "mutation" to the duration attributes of the agent's activities (12). The router is a time-dependent best path algorithm, normally using as link costs the link travel times from the previous iteration (13).

Mode choice will not be simulated by a module per se, but instead by giving every agent both a car and a non-car plan. Further details will be described later.

One plan is marked as "selected." The traffic flow simulation executes all agents' selected plans simultaneously on the network and provides output describing what happened to each individual agent during the execution of its plan. The traffic flow simulation is implemented as a queue simulation, in which each street (link) is represented as a first-in first-out queue with two restrictions $(14,15)$. First, each agent has to remain for a certain time on the link, corresponding to the free-speed travel time. Second, a link storage capacity is defined that limits the number of agents on the link. If it is filled up, no more agents can enter that link.

The modules base their decisions on the output of the traffic flow simulation (e.g., knowledge of congestion) using feedback from the multiagent simulation structure $(16,17)$. This sets up an iteration cycle that runs the traffic flow simulation with specific plans for the agents and then uses the planning modules to update the plans; these changed plans are again fed into the traffic flow simulation, and so on, until consistency between modules is reached. The feedback cycle is controlled by the agent database, which also keeps track of multiple plans generated by each agent.

Ten percent of the agents generate new plans by taking an existing plan, making a copy of it, and then modifying the copy with the time adaptation or the router module. The other agents reuse one of their existing plans. The probability to change the selected plan is calculated by a model, which in the steady state converges to a logit model:

$p_{j}=\frac{e^{\beta \cdot s_{j}}}{\sum_{i} e^{\beta \cdot s_{i}}}$

where

$$
\begin{aligned}
p_{j} & =\text { probability for plan } j \text { to be selected } \\
s_{j} & =\text { its current score }, \text { and } \\
\beta & =\text { sensitivity parameter, set to } 2 .
\end{aligned}
$$

The repetition of the iteration cycle coupled with the agent database enables the agents to learn how to improve their plans over many iterations. Because the number of plans that one agent may have is limited by memory constraints, the plan with the worst performance is deleted when a new plan is added to a person who already has the maximum number of plans permitted. The iteration cycle continues until the system has reached a relaxed state. At this point, there is no quantitative measure of when the system is "relaxed"; the cycle is simply allowed to continue until the outcome is stable.

\section{Scoring Plans}

To compare plans, it is necessary to assign a quantitative score to the performance of each plan. In this work, to be consistent with economic appraisal, a simple utility-based approach is used. The elements of the approach are as follows:

- The total score of a plan is computed as the sum of individual contributions:

$U_{\mathrm{total}}=\sum_{i=1}^{n} U_{\mathrm{perf}, i}+\sum_{i=1}^{n} U_{\mathrm{late}, i}+\sum_{i=1}^{n} U_{\mathrm{tr}, i}$

where

$$
\begin{aligned}
U_{\mathrm{total}}= & \text { total utility for a given plan; } \\
n= & \text { number of activities, which equals the number of trips } \\
& \text { (the first and the last activity—both "home"-are counted } \\
& \text { as one) } \\
U_{\text {perf }, i}= & \text { (positive) utility earned for performing activity } i \\
U_{\text {late }, i}= & \text { (negative) utility earned for arriving late to activity } i \text {; and } \\
U_{\mathrm{tr}, i}= & \text { (negative) utility earned for traveling during trip } i .
\end{aligned}
$$

To work in plausible real-world units, utilities are measured in euros $(€)$.

- A logarithmic form is used for the positive utility earned by performing an activity:

$U_{\mathrm{perf}, i}\left(t_{\mathrm{perf}, i}\right)=\beta_{\mathrm{perf}} \cdot t_{*, i} \cdot \ln \left(\frac{t_{\mathrm{perf}, i}}{t_{0, i}}\right)$

where

$t_{\text {perf }}=$ actual performed duration of the activity,

$t_{*}=$ "typical" duration of an activity, and

$\beta_{\text {perf }}=$ marginal utility of an activity at its typical duration.

$\beta_{\text {perf }}$ is the same for all activities, because in equilibrium all activities at their typical duration need to have the same marginal utility.

- $t_{0, i}$ is a scaling parameter that is related to the minimum duration and to the importance of an activity. As long as dropping activities from the plan is not allowed, $t_{0, i}$ has essentially no effect.

- The (dis)utility of being late is uniformly assumed as

$U_{\text {late }, i}=\beta_{\text {late }} \cdot t_{\text {late }, i}$

where $\beta_{\text {late }}$ is the marginal utility (in $€ / \mathrm{h}$ ) for being late, and $t_{\text {late }, i}$ is the number of hours late to activity $i$. $\beta_{\text {late }}$ is usually negative.

- The (dis)utility of traveling is uniformly assumed as

$U_{\mathrm{tr}, i}=\beta_{\mathrm{tr}} \cdot t_{\mathrm{tr}, i}$

where $\beta_{\mathrm{tr}}$ is the marginal utility (in $€ / \mathrm{h}$ ) for travel, and $t_{\mathrm{tr}, i}$ is the number of hours spent traveling during trip $i$. $\beta_{\text {tr }}$ is usually negative. 
In principle, arriving early or leaving early could also be punished. There is, however, no immediate need to punish early arrival, because waiting times are already indirectly punished by forgoing the reward that could be accumulated by doing an activity instead (opportunity cost). In consequence, the effective (dis)utility of waiting is already

$$
\frac{-\beta_{\mathrm{perf}} t_{*, i}}{t_{\mathrm{perf}, i}} \approx-\beta_{\mathrm{perf}}
$$

Similarly, that opportunity cost has to be added to the time spent traveling, arriving at an effective (dis)utility of traveling of

$$
\frac{-\left|\beta_{\mathrm{tr}}\right|-\beta_{\text {perf }} t_{*, i}}{t_{\mathrm{perf}, i}} \approx-\left|\beta_{\mathrm{tr}}\right|-\beta_{\text {perf }}
$$

No opportunity cost needs to be added to late arrivals because the late arrival time is spent somewhere else. In consequence, the effec-

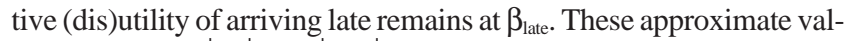
ues $\left(\beta_{\text {perf }}, \beta_{\text {perf }}+\left|\beta_{\text {tr }}\right|\right.$, and $\left.\left|\beta_{\text {late }}\right|\right)$ are the values that would correspond to the consensus values of the Vickrey model parameters (18).

\section{MODE CHOICE MODEL}

The basic idea behind the mode choice model is that each agent always has at least one car plan and one noncar plan. Apart from that, plans are treated as described earlier. Because this always keeps both modes in the choice set, a decision between plans according to Equation 1 is also a choice between modes.

That requires changes in many parts of the simulation framework, namely, the transport simulation, the scoring of plans, and the replanning. These changes are described in the following.

\section{Generating Noncar Plans}

To generate noncar plans, an initial demand with car plans must exist already. Starting with that initial demand, the leg modes of all legs in each plan are set to "car," and the fastest routes are calculated. Then, each plan is duplicated, changing all leg modes in the duplicated plans to "noncar."

Although no exact route is provided, the duration of every noncar trip is assumed to take twice as long as the car mode at free speed. This is based on the (informally stated) goal of the Berlin public transit company to generally achieve door-to-door travel times that are no longer than twice as long as car travel times. This, in turn, is based on the observation that noncaptive travelers can be recruited into public transit when it is faster than this benchmark (19). For the purposes of this paper, it is assumed that all noncar modes very roughly have the shared characteristics that they are slower than the (noncongested) car mode- this will be further disaggregated in future work. In the same vein, for both car and noncar trips there are no separate considerations of access and egress.

\section{Handling Noncar Plans in Transport Simulation}

Currently, the simulation supports no walk or rail network, only a road network. Thus, only car legs can be truly simulated. Agents on noncar legs are teleported from one location to the next. But the teleportation is not instantaneous; it takes some amount of time, which can be stored in the legs as planned travel duration. Although this does not impose any transit vehicle capacity constraints, it would still allow having individual travel times, depending on agents' demographics or chosen noncar mode (e.g., bike, walk, transit). The simulation still generates departure and arrival events for noncar legs, which can be used for analyses.

\section{Scoring Noncar Plans}

The scoring of noncar plans is very similar to the scoring of car plans as described previously, only the marginal disutility of traveling changes. This is expressed by using $\beta_{\mathrm{tr}, \mathrm{nc}}$ for the marginal utility of traveling, instead of $\beta_{\mathrm{tr}, \mathrm{car}} \cdot \beta_{\mathrm{tr}, \mathrm{car}}$ and $\beta_{\mathrm{tr}, \mathrm{nc}}$ are not values of time by themselves, but they are additional marginal disutilities caused by traveling, in addition to the opportunity cost of time. This is consistent with econometric approaches (20).

\section{Replanning with Noncar Plans}

During replanning, plans are duplicated and modified (see "iteration cycle" in the section on simulation structure). This also holds for noncar plans. The only difference is that the plans deletion module makes sure that at least one plan of every mode is kept for every agent. This is to make sure that all agents keep their ability to change mode until the end of the iterations.

The steps above integrate mode choice into the replanning process that takes place iteratively with the simulation. Instead of precalculation of the mode choice before the traffic assignment as done in the traditional four-step process, mode choice is now treated at the same level as route choice in the traffic assignment.

\section{TEST SCENARIO}

\section{Network}

To test the mode choice model, a simple test network was used (see Figure 1), consisting only of a cycle of one-way links (a simplified version of another test network used internally, which explains the numbering of the links). Traffic runs clockwise, that is, agents have their home location at Link 1 and work on Link 20. The capacity of all links except Links 6 and 15 are (unrealistically) high so as to minimize the influence these links have on the traffic, essentially making it possible for most agents to drive with free speed. Links 6 and 15 have reduced capacity, building a bottleneck.

\section{Initial Plans}

The synthetic population consists of 2,000 agents. All agents have their home activity at Link 1, which they initially leave at 6:00 a.m. They drive to work (located on Link 20) with a car via Links 6 and 15, where they stay for $8 \mathrm{~h}$, after which they drive back home to Link 1 via Links 21 through 23 . The free-speed travel time from Link 1 to Link 20 is $15 \mathrm{~min}$. The free-speed travel time from Link 20 to Link 1 is $39 \mathrm{~min}$. Thus the total free-speed travel time driving by car is $54 \mathrm{~min}$ or $0.9 \mathrm{~h}$.

Because the agents are forced to remain on that route, the scenario converts into the well-known Vickrey bottleneck scenario $(18,21)$; see below for more details.

In addition, each agent possesses an initially nonactive plan that uses the noncar mode for both trips. These trips take twice as long as by car in an empty network, that is, 30 min from Link 1 to Link 20, 


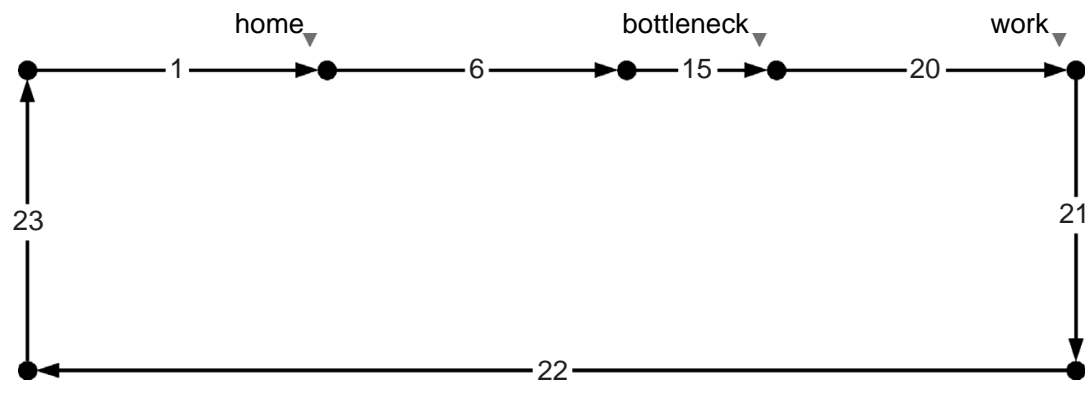

\begin{tabular}{|l|r|r|r|}
\hline Links & Length $(\mathrm{m})$ & $\begin{array}{r}\text { Freespeed Velocity } \\
(\mathrm{m} / \mathrm{s})\end{array}$ & Capacity $(\mathrm{veh} / \mathrm{h})$ \\
\hline \hline $1,20,21,23$ & 10,000 & 27.78 & 36,000 \\
\hline 22 & 35,000 & 27.78 & 36,000 \\
\hline 6 & 10,000 & 27.78 & 3,600 \\
\hline 15 & 5,000 & 27.78 & 1,000 \\
\hline
\end{tabular}

FIGURE 1 Links of test network with their corresponding IDs and attributes.

and 78 min from Link 20 to Link 1. The total noncar travel time is $108 \mathrm{~min}$ or $1.8 \mathrm{~h}$. In contrast to the car travel times, these noncar travel times are not affected by congestion. The first trip starts at 6:30 a.m., so the agents will arrive exactly at 7:00 a.m. at their workplaces.

\section{Behavioral Parameters}

The behavioral parameters are set and can be interpreted as follows:

- Utility of performing an activity at its typical duration is $\beta_{\text {perf }}=$ $6 € / \mathrm{h}$.

- Marginal disutility of coming late is $\beta_{\text {late }}=-18 € / \mathrm{h}$.

- Additional marginal disutility of traveling with a car is $\beta_{\mathrm{tr}, \mathrm{car}}=$ $-6 € / \mathrm{h}$.

- Additional marginal disutility of traveling with noncar mode is $\beta_{\text {tr,nc }} \in[-10,-9, \ldots,+2]$ (see below).

- Constant in binary logit model (Equation 1) is $\beta=2$.

- "Typical" durations of $t_{*, w}=8$ and $t_{*, h}=12 \mathrm{~h}$ for work and home mean that work and home times have a tendency to arrange themselves with a ratio of 8:12 (i.e., 2:3): assume a fixed travel time budget. In this situation, for optimality of the scoring function the marginal utilities of duration, $\partial U_{\mathrm{per}, i} / \partial t_{\mathrm{per}, i}=\beta_{\mathrm{perf}} t_{*, i} / t_{\mathrm{perf}, i}$, need to be equal for all activity types, resulting in

$\frac{t_{h}}{t_{*, h}}=\frac{t_{w}}{t_{*, w}}$

The result is only approximately correct when the overall travel time varies.

- A work start exactly at 7:00 a.m. means that $(a)$ no utility can be accumulated from an arrival earlier than 7:00 a.m. and (b) any late arrival is immediately punished with $\beta_{\text {late }}=-18 € / \mathrm{h}$. Because of the argument made earlier concerning the opportunity cost of forgone activity time in situation $(a)$, the effective marginal disutility of early arrival is

$$
\frac{-\beta_{\text {perf }} t_{*, i}}{t_{\text {perf }, i}} \approx-\beta_{\text {perf }}=-6 € / \mathrm{h}
$$

Because the effective marginal disutility of car traveling is, by the same argument,

$$
\frac{-\beta_{\text {perf }} t_{*, i}}{t_{\text {perf }, i}} \approx-\beta_{\text {perf }}-\left|\beta_{\mathrm{tr}, \mathrm{car}}\right| \approx-12 € / \mathrm{h}
$$

the effective values of time of this study are approximately the same as the consensus values of $(-6,-12,-18)$ of the Vickrey scenario $(18$, $21)$. The return trip has no influence because there is no congestion.

\section{Simulation Results}

The simulation in the test setup was run with different values for $\beta_{\mathrm{tr}, \mathrm{nc}}$, resulting in different mode shares. Each simulation was first run for 1,000 iterations. In each iteration, $10 \%$ of the agents were modified by the time allocation module; all other agents chose an existing plan. After that, the simulation was continued for 100 more iterations, but without time adaptation. This allowed agents to select their best plan, no longer being forced to execute (possibly bad) plans after replanning.

$\beta_{\mathrm{tr}, \mathrm{nc}}$ was varied from +2 to -10 in increments of -1 . Figure 2 shows the resulting car mode shares as dots. It can be clearly seen that an increase of the marginal disutility of traveling in the noncar mode leads to an increasing number of agents choosing car as the transportation mode. In the following section, these results are validated by comparing them with the theoretical values one should expect based on the aforementioned mode choice model.

\section{THEORETICAL CALCULATIONS}

Because of the simulation setup, the mode share of the car mode, $f_{\text {car }}$, follows a binary logit model. This statement is, in fact, correct only when the number of car plans is equal to the number of noncar plans for every agent. (See the end of the section for a comment on this)

$f_{\text {car }}=\frac{\exp \left(\beta \cdot U_{\text {car }}\left(f_{\text {car }}\right)\right)}{\exp \left(\beta \cdot U_{\text {car }}\left(f_{\text {car }}\right)\right)+\exp \left(\beta \cdot U_{\text {nc }}\right)}$ 


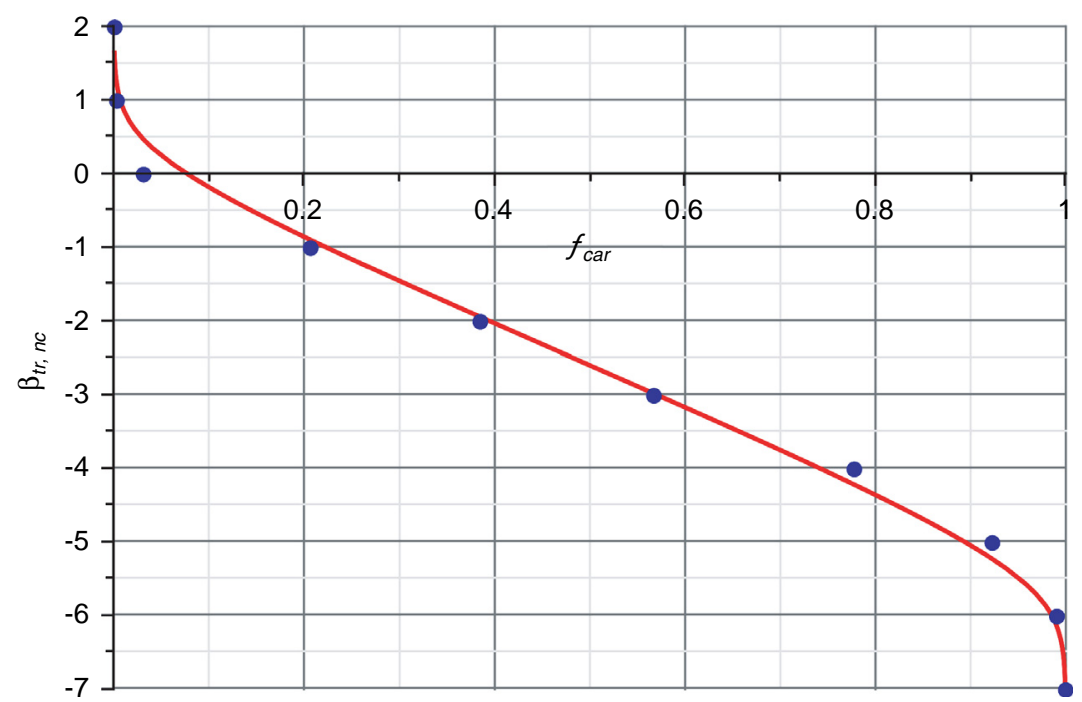

FIGURE 2 Noncar travel marginal disutilities ( $\boldsymbol{\beta}_{\mathrm{tr}, \mathrm{nc}}$ ) for different car shares $\left(f_{\text {car }}\right.$ ). The line refers to the analytical result, the dots to the simulation results.

$U_{\text {car }}$ and $U_{\text {nc }}$ are the total utilities of agents traveling either with a car or using the noncar transport mode. These are utilities for the full daily plan, and not partial utilities for the mode choice contribution only. These utilities are defined according to Equation 2, with only the two activities "home" and "work":

$$
\begin{aligned}
U_{\text {mode }}= & \beta_{\text {perf }} \cdot t_{*, h} \cdot \ln \left(\frac{t_{h, \text { mode }}}{t_{0, h}}\right)+\beta_{\text {perf }} \cdot t_{*, w} \cdot \ln \left(\frac{t_{w, \text { mode }}}{t_{0, w}}\right) \\
& +\beta_{\text {tr }, \text { mode }} \cdot t_{\mathrm{tr} \text {,mode }}+\beta_{\text {late }} \cdot t_{\text {late }}
\end{aligned}
$$

As mentioned before, travel times depend on the transport mode.

\section{Noncar Mode}

Taking the activity duration ratio of Equation 6 together with the time budget equation $t_{h, \mathrm{nc}}+t_{w, \mathrm{nc}}+t_{\mathrm{tr}, \mathrm{nc}}=24 h$, one obtains the following for people using the noncar mode:

$t_{h, \mathrm{nc}}=\left(24 h-t_{\mathrm{tr}, \mathrm{nc}}\right) \cdot \frac{t_{*, h}}{t_{*, h}+t_{*, w}}$

$t_{w, \mathrm{nc}}=\left(24 h-t_{\mathrm{trn \textrm {nc }}}\right) \cdot \frac{t_{*, w}}{t_{*, w}+t_{*, h}}$

At this point, all variables for Equation 8 for the noncar mode, assuming on-time arrival, are expressed in the parameters of the simulation.

\section{Car Mode}

For car users, the calculation is more complex. In line with Arnott et al. (18) and Vickrey (21), it will be assumed that at the end of the day every agent will have experienced the same total utility: Although some may spend more time traveling (by being stuck in a traffic jam) but arrive at the right time at the workplace, other agents may decide to leave early, traveling the whole route with free speed, but also arrive at work early, forgoing any utility by performing an activity because the workplace is still closed. Other agents again may stay longer at home, traveling after the jam has disappeared, arriving late at work, and receiving the schedule delay penalty for that. One can obtain results by looking just at the first and the last agent to arrive at work. When Equation 8 is equated for these two, the travel time drops out because it is the same for both, and one arrives at

$$
\begin{aligned}
\beta_{\text {perf }} \cdot t_{*, h} \cdot \ln ( & \left.\frac{t_{h, \mathrm{car}}-\tau_{h}}{t_{0, h}}\right)+\beta_{\mathrm{perf}} \cdot t_{*, w} \cdot \ln \left(\frac{t_{w, \mathrm{car}}-\tau_{w}}{t_{0, w}}\right) \\
= & \beta_{\mathrm{perf}} \cdot t_{*, h} \cdot \ln \left(\frac{t_{h, \mathrm{car}}}{t_{0, h}}\right)+\beta_{\text {perf }} \cdot t_{*, w} \cdot \ln \left(\frac{t_{w, \mathrm{car}}}{t_{0, w}}\right)+\beta_{\text {late }} \cdot t_{\text {late }}
\end{aligned}
$$

where the left-hand side refers to the person who arrives early and who suffers $\tau_{h}, \tau_{w}$ reductions of his or her activity durations. After linearization and dropping of terms that cancel out, this becomes

$$
-\tau_{h} \cdot \beta_{\text {perf }} \cdot t_{*, h} \cdot \frac{1}{t_{h, \mathrm{car}}}-\tau_{w} \cdot \beta_{\text {perf }} \cdot t_{*, w} \cdot \frac{1}{t_{w, \mathrm{car}}} \approx \beta_{\text {late }} \cdot t_{\text {late }}
$$

From the optimal time allocation, Equation 6, one infers that also for the time deductions $\tau_{h}, \tau_{w}$ one needs $\tau_{h} / \tau_{w}=t_{*, h} / t_{*, w}$ and therefore $\tau_{h}=t_{\text {early }} \cdot t_{*, h} /\left(t_{*, h}+t_{*, w}\right)$ and $\tau_{w}=t_{\text {early }} \cdot t_{*, w} /\left(t_{*, h}+t_{*, w}\right)$. Taking this and once more Equation 6 directly, one obtains, after some algebra,

$t_{\text {early }} \beta_{\text {perf }} \frac{t_{*, h}}{t_{h, \text { car }}} \approx\left|\beta_{\text {late }}\right| t_{\text {late }}$

In addition, one has the equation for the bottleneck

$t_{\text {early }}+t_{\text {late }}=\frac{|A| \cdot f_{\text {car }}}{C_{b}}$

where

$|A|=$ total number of agents,

$C_{b}=$ flow capacity of bottleneck, and

$f_{\text {car }}=$ share of car users. 
The equation states that the capacity of the bottleneck is exactly enough to serve all agents between the first and the last. Inserting Equation 11, one obtains

$t_{\text {early }} \approx \frac{\left|\beta_{\text {late }}\right| t_{h, \text { car }}}{\left|\beta_{\text {late }}\right| t_{h, \text { car }}+\beta_{\text {perf }} t_{* h}} \cdot \frac{|A| \cdot f_{\text {car }}}{C_{b}}$

Similar to Equations 9 and 10, the optimal activity durations for the "early" agent are

$t_{h, \mathrm{car}}+t_{w, \mathrm{car}}+t_{\mathrm{tr}, \mathrm{fs}}+t_{\text {early }}=24 h$

$t_{h, \mathrm{car}}=\left(24 h-t_{\mathrm{tr}, \mathrm{fs}}-t_{\mathrm{early}}\right) \cdot \frac{t_{*, h}}{t_{*, h}+t_{*, w}}$

$t_{w, \text { car }}=\left(24 h-t_{\mathrm{tr}, \mathrm{fs}}-t_{\mathrm{early}}\right) \cdot \frac{t_{*, w}}{t_{*, w}+t_{*, h}}$

where $t_{\mathrm{tr}, \mathrm{fs}}$ is the free-speed travel time by car. Substituting $t_{h, \text { car }}$ from Equation 14 into Equation 13 leads to an equation that contains only $t_{\text {early }}$ and $f_{\text {car }}$ as unknowns. It can be seen that the resulting equation contains the square of $t_{\text {early }}$. Solving that resulting equation provides two solutions for $t_{\text {early }}$, of which only one is useful because the other leads to negative times for either $t_{\text {early }}$ or $t_{\text {late }}$ in Equation 12 . Thus at this point one knows $t_{\text {early }}$ and in consequence $t_{h \text {,car }}$ and $t_{w, \text { car }}$ as functions of $f_{\text {car }}$. The expressions can be written down, but are rather long and not easy to interpret.

\section{Complete Mode Choice}

Recall that the interest here lies in an expression that relates the mode share, $f_{\text {car }}$, and the additional marginal disutility of the noncar mode, $\beta_{\mathrm{tr}, \mathrm{nc}}$. What one has at this point is

- The utility of the optimal noncar plan can be computed as a function of $\beta_{\mathrm{tr}, \mathrm{nc}}$ and

- The utility of the optimal car plan can be computed as a function of $f_{\text {car }}$.

What remains is to insert these expressions into Equation 7, which can also be written as

$U_{\text {car }}=\frac{1}{\beta} \cdot \ln \left(\frac{f_{\text {car }}}{1-f_{\text {car }}}\right)+U_{\text {nc }}$

Substituting $U_{\text {car }}$ and $U_{\text {nc }}$ with Equation 8, one obtains

$$
\begin{aligned}
\beta_{\mathrm{perf}} & \cdot t_{*, h} \cdot \ln \left(\frac{t_{h, \mathrm{car}}}{t_{0, h}}\right)+\beta_{\mathrm{perf}} \cdot t_{*, w} \cdot \ln \left(\frac{t_{w, \mathrm{car}}}{t_{0, w}}\right)+\beta_{\mathrm{tr}, \mathrm{car}} \cdot t_{\mathrm{tr}, \mathrm{ss}} \\
= & \frac{1}{\beta} \cdot \ln \left(\frac{f_{\mathrm{car}}}{1-f_{\mathrm{car}}}\right)+\beta_{\mathrm{perf}} \cdot t_{*, h} \cdot \ln \left(\frac{t_{h, \mathrm{nc}}}{t_{0, h}}\right)+\beta_{\mathrm{perf}} \cdot t_{*, w} \\
& \cdot \ln \left(\frac{t_{w, \mathrm{nc}}}{t_{0, w}}\right)+\beta_{\mathrm{tr}, \mathrm{nc}} \cdot t_{\mathrm{tr}, \mathrm{nc}}
\end{aligned}
$$

Recall that for the car mode the "first" (= most early) agent is being considered; the term concerning late arrival is thus dropped.

More variables can be substituted in Equation 17 by their corresponding calculations in the previous equations. Although it could still be solved analytically, it once more becomes quite complex and not easily readable.

Evidently, $\beta_{\mathrm{tr}, \mathrm{nc}}$ can be isolated in Equation 17, but not so $f_{\text {car }}$ if one remembers that $f_{\text {car }}$ is also part of $t_{\text {early }}$, which is used to substitute $t_{h, \text { car }}$ and $t_{w, \text { car }}$ (Equations 13-15).

Extracting $\beta_{\mathrm{tr}, \mathrm{nc}}$ and plotting it as a function of $f_{\text {car }}$ ranging from 0 to 1 , one obtains the line shown in Figure 2. Comparing it with the simulation (dots), one can see that the results are very similar. Only small variations can be seen, most likely due to the discrete size of agents in the simulation as well as the not completely predictable behavior of random numbers used in the simulation. In addition, the calculations assume that every agent has an optimal plan, which cannot be guaranteed in the simulation.

The fact that, despite the noise, the mode choice curve is "steeper" in the simulations than in the analytical calculations is due to the learning algorithm: If for an agent one mode is clearly better than the other mode, the better mode will have more plans. This gives an additional statistical advantage to the better mode, making the curve steeper.

Overall, the mode choice model is found to be in excellent agreement with the theoretical calculations. This, on the one hand, verifies the implementation of the model. On the other hand, it means that, to an extent, it is possible to understand analytically what the simulation does, which will help to uncover and allow an understanding of the economic and behavioral principles embedded in the implementation.

\section{LARGE-SCALE APPLICATION}

The mode choice model was also applied to a large-scale, real-world scenario. The area of Zurich, Switzerland, which has about 1 million inhabitants, was used for this application. The following paragraphs give a simplified description of the scenario. A full description of the scenario can be found in Chen et al. (22).

The network is a Swiss regional planning network that includes the major European transit corridors. It consists of 24,180 nodes and 60,492 links.

The simulated demand consists of all travelers in Switzerland that are inside an imaginary boundary around Zurich at least once during their day $(22,23)$. All agents have complete day plans with activities, such as home, work, education, shopping, and leisure, based on microcensus information $(24,25)$. The time window during which activities could be performed was limited to certain hours of the day: work and leisure could be performed from 06:00 to 20:00, shopping from 08:00 to 20:00, whereas home and leisure had no restrictions. Unlike the sample scenario described in the sections above, there was no punishment for being late. That was not possible because agents could split their work activity into two or more parts, for example, one in the morning and one in the afternoon. In such a case it would be complicated to specify when an agent starts an activity late.

To speed up computations, a random $10 \%$ sample consisting of 181,725 agents was chosen from the synthetic population for simulation. In this large-scale application, the agents not only could perform time adaptation as described previously, but could also do route adaptation, which is essential for the car mode. For comparison, the same scenario was run with the precalculated mode choice [see Chen et al. (22)].

Simulated traffic volumes were compared with the hourly traffic volumes from 159 real-world counting stations. Figure 3 shows the mean relative error between hourly flows in reality and hourly flows from the simulation. Figure $3 a$ contains the result from the fixed, 


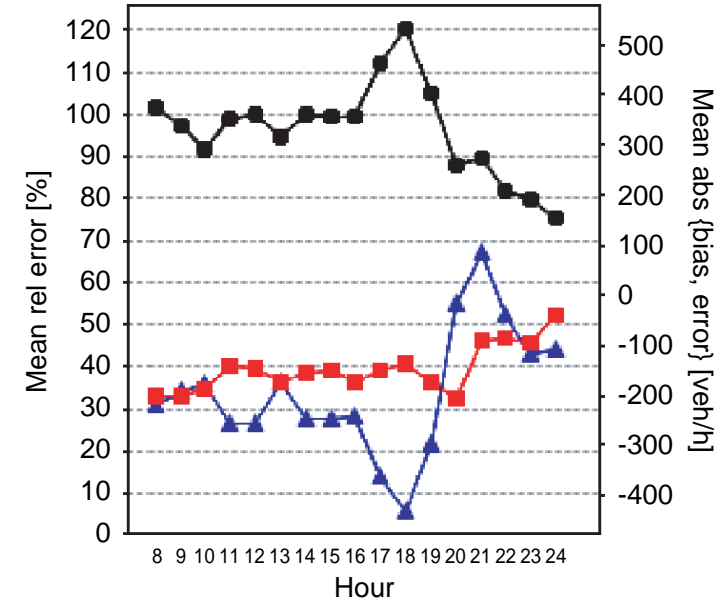

- Mean rel error $\rightarrow$ Mean abs error $\leftarrow$ Mean abs bias

(a)

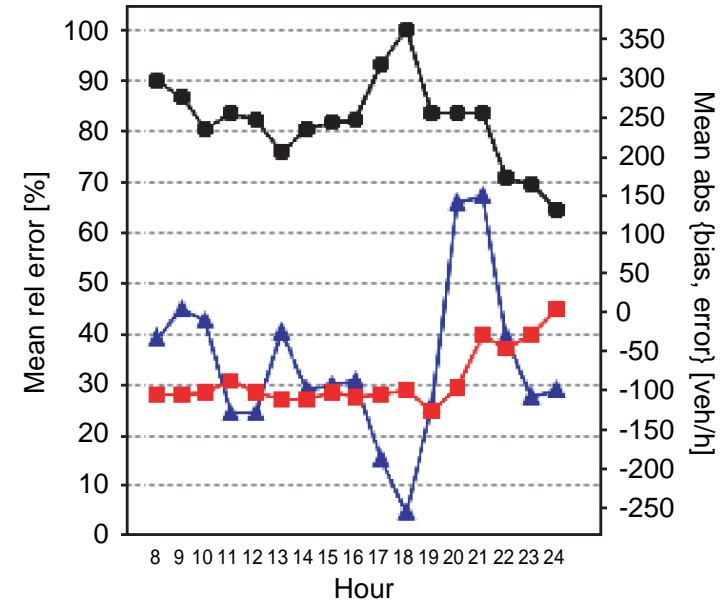

Mean rel error

Mean abs error $₫$ Mean abs bias

(b)

FIGURE 3 Comparison of simulated traffic volumes with real-world counts. Note different scales on $y$-axis: (a) precalculated mode choice and $(b)$ mode choice during simulation $\left(\beta_{\mathrm{tr}, \mathrm{nc}}=-3\right)$.

predetermined mode choice; Figure $3 b$ contains the result of the new adaptive mode choice that was explained in this paper. One notices a quite distinct reduction in the average error, from about $40 \%$ to about $30 \%$. Also the absolute bias, in blue, is reduced.

For the large-scale tests, the marginal disutility for the car mode, $\beta_{\mathrm{tr}, \mathrm{car}}$, was set to $-6 € / \mathrm{h}$; the marginal disutility for the noncar mode, $\beta_{\mathrm{tr}, \mathrm{nc}}$, was varied between 0 and -6 . An interpretation of this might be that measures are discussed that change the attractiveness of the noncar modes, leaving everything else, including the travel times, constant. An obvious concrete example would be fare changes. And the importance of the results at this point is not so much the magnitude of the response itself, but the fact that the model displays the interaction between activity timing and mode choice. Figure 4 shows the number of agents en route with cars over the time of day. It can be clearly seen that the number of car users decreases the lower the travel marginal disutility for the noncar mode becomes. The peaks at 6 a.m. and 8 p.m. are due to the opening time restrictions.

Figure $5 a$ shows all departures as a function of the time of day, for different values of $\beta_{t r, n c}$. Because demand itself is inelastic, the area under all the curves is the same. One notices, however, a shift toward the peak periods when the marginal disutility of the noncar mode is reduced. That occurs because there is no peak avoidance in the noncar mode and because the peak period becomes less congested, meaning that other car users readjust their schedules toward the peak hour.

Noncar departures (Figure $5 b$ ) show the expected behavior: more noncar departures at all times as a function of a reduced marginal disutility.

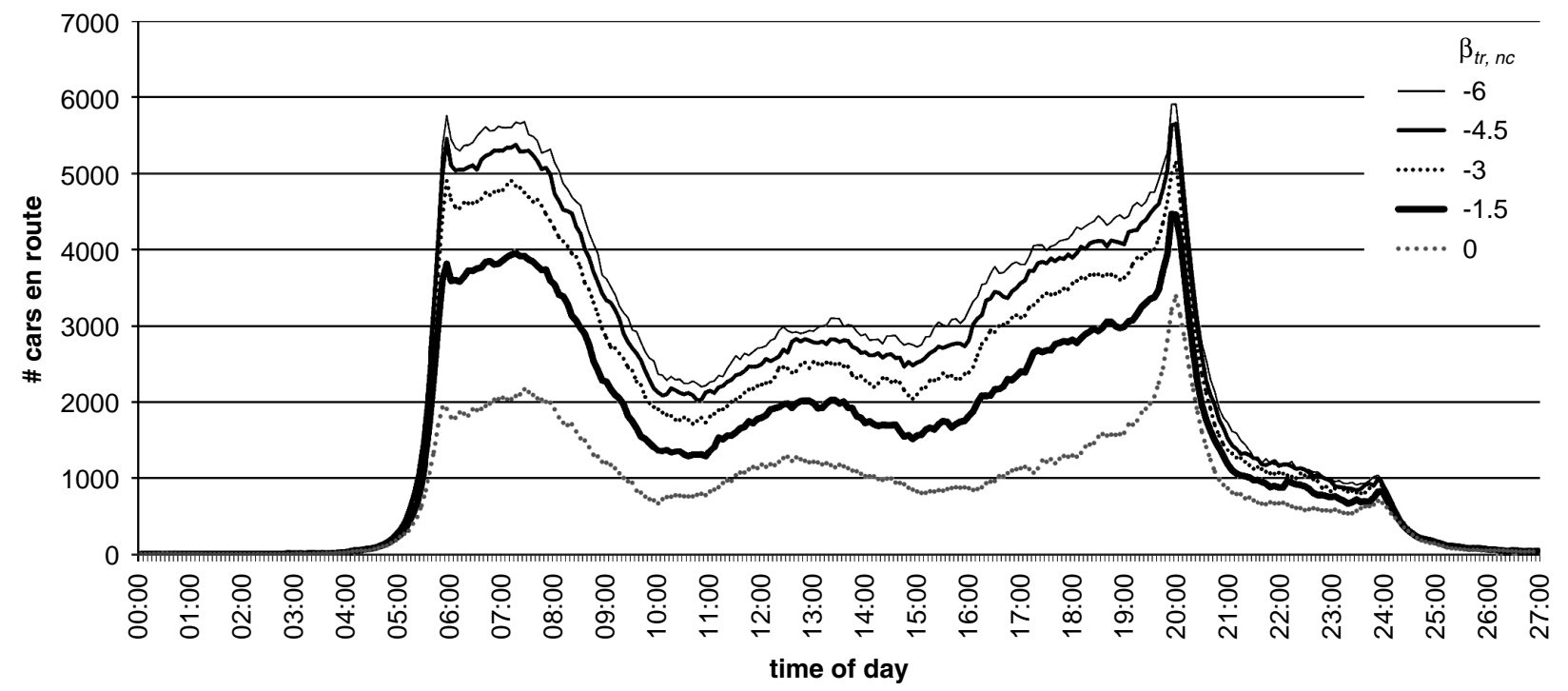

FIGURE 4 Cars en route in large-scale scenario over time of day with different marginal disutilities for traveling with noncar modes. 


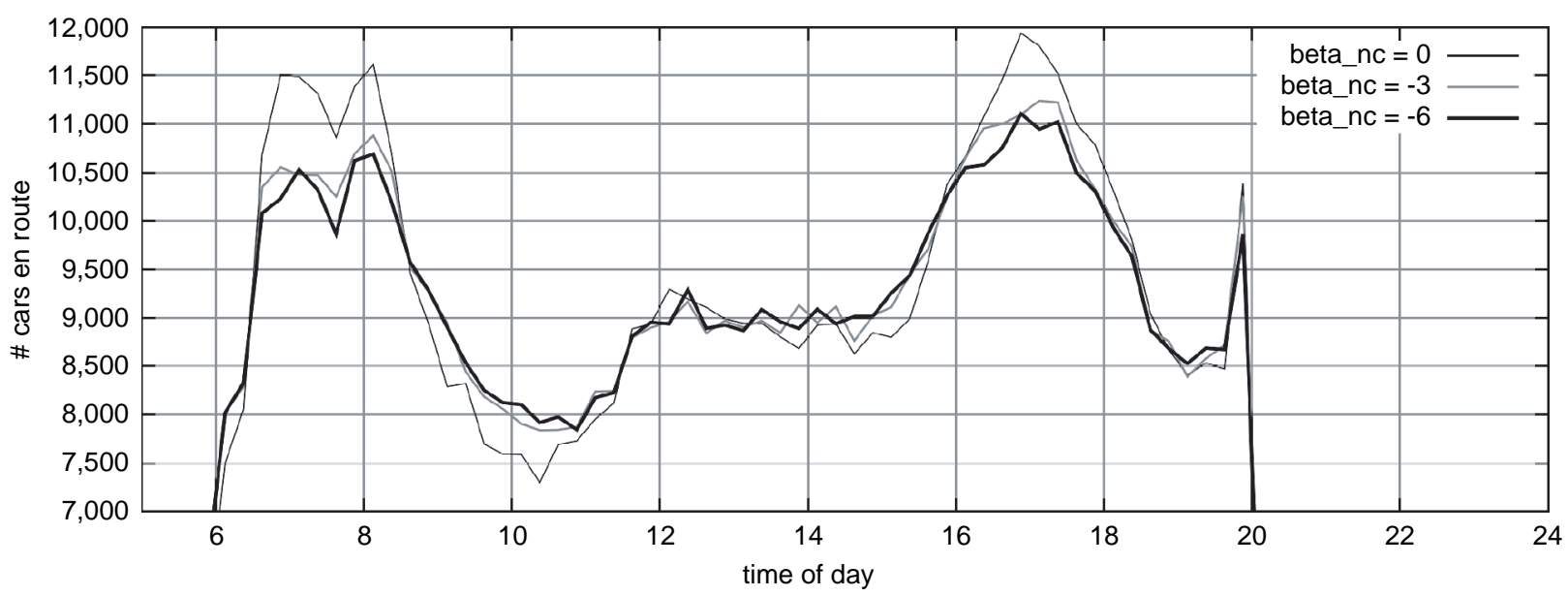

(a)

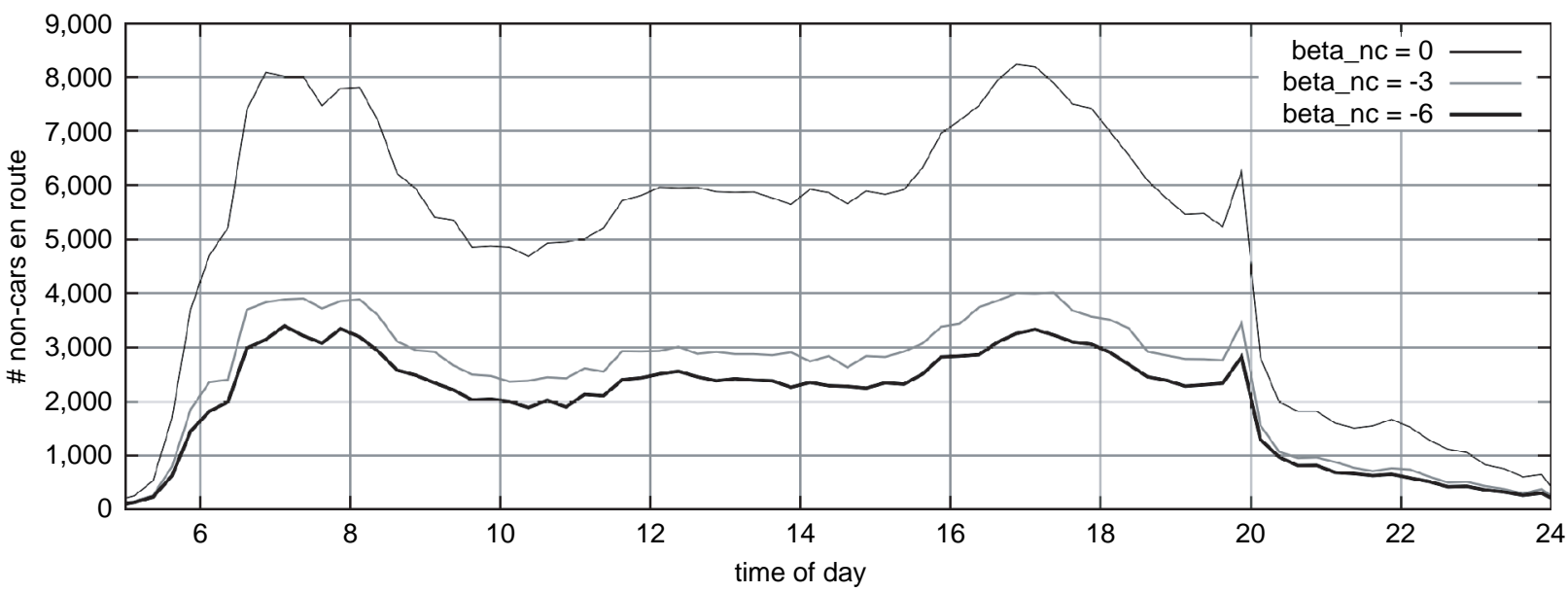

(b)

FIGURE 5 Number of agents departing per 15 min over time of day: (a) all agents departing and ( $b$ ) agents departing with noncar mode.

Outside the time from 6 a.m. to 6 p.m., the noncar share is markedly lower than during the day. The reason is that during those times there is little car congestion, thus making the car more attractive.

\section{FURTHER STEPS}

At the moment, only one transport mode can be used for the complete plan. That is, all trips of a given day need to be done by the same mode. Although the data structures, file formats, and simulation could deal with a different transport mode per leg, there are some conceptual points that it is desirable to solve first before applying mode choice to a subtour level.

The simulation setup would allow having different $\beta_{\text {tr.nc }}$ over the time of day, as every trip has a departure time. This could be used to model a changing attractiveness to use the noncar modes during a day. One example might be to improve the quality of service in transit in the late evening or night hours, resulting in a lower absolute marginal disutility during that time of day.

An improved router for noncar modes would improve results. Possibilities are the use of transit schedules instead of the "double free speed travel time" assumption currently used. Currently, that assumption makes the noncar mode highly unattractive for longdistance trips. This will most likely change by the use of more realistic travel times, especially for long-distance trips that are served well by fast trains.

The simulation should not only teleport agents with noncar mode, but also actually simulate them as well. Different aspects of this would be important to include, say, public transport vehicle overcrowding effects or the effect of public transport being caught in car congestion. It would require adding transit vehicles, bikes, and other means of transport, together with their characteristics, schedules, and so on.

A car ownership model, or arguably a lifestyle model, could be added in the demand modeling. That would reduce the choice between car and noncar mode to travelers that actually have access to a car. A preliminary attempt to do this for the Zurich scenario did not lead to improved results with respect to the real-world traffic counts. This was presumably a result of the fact that the car ownership model was based on zonal characteristics, whereas the mode choice model of the present simulation, at least on the car side, picks up very detailed accessibility issues. It becomes quite clear that the behavioral basis of all relevant decision models needs to be consistent. 


\section{CONCLUSION}

It was shown how to include a noncar mode into a multiagent transport simulation with relatively few conceptual changes. The noncar mode was integrated by giving every agent two initial plans, one using the car for all trips and one using the noncar mode for all trips. The noncar trips are assumed to use twice as much travel time as the uncongested car mode. Travelers can then, in the simulation, adjust times and car routes; the performance of the resulting plans is scored after execution in the traffic flow simulation, based on a utility function that includes positive utility for performing an activity, different negative utilities for traveling by different modes, and opening times outside which no utility for performing an activity can be accumulated.

The model was first tested in a simplified scenario based on the famous Vickrey bottleneck example. The noncar mode was used as an alternative to the congested car mode. It was shown that the analytical calculation and the simulation model produce the same results when one looks at the mode split as a function of the noncar mode marginal disutility.

The model was then applied to a realistic real-world example for the Zurich metropolitan area. The reaction of users to changes in the noncar marginal disutility was analyzed in some detail, including temporal reactions. Adding the mode choice to the large-scale scenario improved the realism of the scenario when the simulated traffic volumes were compared to data from counting stations.

\section{ACKNOWLEDGMENTS}

The authors thank Michael Balmer for his contributions to the largescale application in the Zurich area. The compute cluster is maintained by the Faculty for Mathematics at the Berlin Institute of Technology.

\section{REFERENCES}

1. Verhoef, E. T. Marginal Cost Based Pricing in Transport. Key Implementation Issues from the Economic Perspective. Presented at Seminar One of IMPRINT-EUROPE, 2001. www.imprint-eu.org/public/EV_V2.PDF.

2. Bonsall, P., J. Shires, D. Ngoduy, H. Link, A. Becker, P. Papaioannou, and P. Xanthopoulos. Optimal Complexity of Prices for Transport Infrastructure. Deliverable 6 of GRACE (generalisation of research on accounts and cost estimation). Funded by 6th framework programme, ITS, University of Leeds, Leeds, United Kingdom, 2007.

3. Ortúzar, J. de D., and L. G. Willumsen. Modelling Transport. Wiley, Chichester, United Kingdom, 1995.

4. Richards, M. G., and M. Ben-Akiva. A Simultaneous Destination and Mode Choice Model for Shopping Trips. Transportation, 1974, pp. 343-356.

5. Jonnalagadda, N., J. Freedman, W. A. Davidson, and J. D. Hunt. Development of Microsimulation Activity-Based Model for San Francisco: Destination and Mode Choice Models. In Transportation Research Record: Journal of the Transportation Research Board, No. 1777, TRB, National Research Council, Washington, D.C., 2001, pp. 25-35.

6. Boyce, D., and H. Bar-Gera. Validation of Multiclass Urban Travel Forecasting Models Combining Origin-Destination, Mode, and Route Choices. Journal of Regional Science, Vol. 43, 2003, pp. 517-540.
7. Lohse, D., G. Bachner, B. Dugge, and H. Teicher. Ermittlung von Verkehrsströmen mit n-linearen Gleichungssystemen unter Beachtung von Nebenbedingungen einschließlich Parameterschätzung. Technical Report 5/1997. Institut für Verkehrsplanung und Straßenverkehr, Dresden, Germany, 1997.

8. Nielsen, O. A., and R. D. Frederiksen. Optimisation of TimetableBased, Stochastic Transit Assignment Models Based on MSA. Annals of Operations Research, Vol. 144, 2006, pp. 263-285.

9. Nökel, K. VISUM 10-Status and Perspectives. Technical report. PTV AG, Karlsruhe, Germany. 2007. www.ptv.de.

10. Zhang, L., and D. Levinson. Agent-Based Approach to Travel Demand Modeling: Exploratory Analysis. In Transportation Research Record: Journal of the Transportation Research Board, No. 1898, Transportation Research Board of the National Academies, Washington, D.C., 2004 pp. 28-36.

11. Raney, B., and K. Nagel. An Improved Framework for Large-Scale Multi-Agent Simulations of Travel Behaviour. In Towards Better Performing European Transportation Systems (P. Rietveld, B. Jourquin, and K. Westin, eds.), Routledge, London, 2006.

12. Balmer, M., B. Raney, and K. Nagel. Adjustment of Activity Timing and Duration in an Agent-Based Traffic Flow Simulation. In Progress in Activity-Based Analysis (H. J. P. Timmermans, ed.), Elsevier, Oxford, United Kingdom, 2005, pp. 91-114.

13. Lefebvre, N., and M. Balmer. Fast Shortest Path Computation in Time-Dependent Traffic Networks. Proc., Swiss Transport Research Conference, Monte Verita, Switzerland. www.strc.ch.

14. Gawron, C. Simulation-Based Traffic Assignment. $\mathrm{PhD}$ thesis. University of Cologne, Cologne, Germany, 1998.

15. Cetin, N., A. Burri, and K. Nagel. A Large-Scale Agent-Based Traffic Microsimulation Based on Queue Model. Proc., Swiss Transport Research Conference (STRC), Monte Verita, Switzerland. www.strc.ch.

16. Kaufman, D. E., K. E. Wunderlich, and R. L. Smith. An Iterative Routing/ Assignment Method for Anticipatory Real-Time Route Guidance. IVHS Technical Report 91-02. Department of Industrial and Operations Engineering, University of Michigan, Ann Arbor, May 1991.

17. Bottom, J. A. Consistent Anticipatory Route Guidance. PhD thesis. Massachusetts Institute of Technology, Cambridge, 2000.

18. Arnott, R., A. de Palma, and R. Lindsey. Economics of a Bottleneck. Journal of Urban Economics, Vol. 27, No. 1, 1990, pp. 111-130.

19. Reinhold, T. Konzept zur integrierten Optimierung des Berliner Nahverkehrs. In Öffentlicher Personennahverkehr. Springer Verlag, Berlin, Germany, 2006.

20. Jara-Díaz, S. R., and R. Guerra. Modeling Activity Duration and Travel Choice from a Common Microeconomic Framework. Proc., Meeting of the International Association for Travel Behavior Research (IATBR), Lucerne, Switzerland, 2003. www.ivt.baug.ethz.ch.

21. Vickrey, W. S. Congestion Theory and Transport Investment. American Economic Review, Vol. 59, No. 2, 1969, pp. 251-260.

22. Chen, Y., M. Rieser, D. Grether, and K. Nagel. Improving a Large-Scale Agent-Based Simulation Scenario. VSP Working Paper 08-15. Transport Systems Planning and Transport Telematics, Berlin Institute of Technology, 2008. www.vsp.tu-berlin.de/publications.

23. Vrtic, M., N. Schüssler, A. Erath, K. Meister, and K. Axhausen. Tageszeitliche Fahrtenmatrizen im Personenverkehr an Werktagen im Jahr 2000. Research report. Swiss Federal Department for Environment, Transport, Energy and Communication, Swiss Federal Office for Spatial Development, Swiss Federal Roads Authority and Swiss Federal Office for Transport, IVT, ETH Zurich, 2007.

24. Eidgenössische Volkszählung. Swiss Federal Statistical Office, Neuchatel, 2000.

25. Ergebnisse des Mikrozensus 2005 zum Verkehr. Swiss Federal Statistical Office, Neuchatel, 2006.

The Transportation Demand Forecasting Committee sponsored publication of this paper. 